InnOvaciOnes de NegOciOs 16(31): 118-142

(c) 2019 UANL, Impreso en México (ISSN: 2007-1191)

Recepción: 3 Marzo de 2018. Aceptación: 22 Mayo de 2018

\title{
Impacto de las capacidades de las TIC que contribuyen a mejorar el desempeño de las MIPyMES (Impact of ITC capabilities that contribute to improve the performance of MSMEs
}

\author{
Jorge Alberto Islas Pineda, Miguel Ángel Palomo González \\ Universidad Autónoma de Nuevo León \\ jorge_islas@msn.com, mapalomo2003@yahoo.com
}

\begin{abstract}
The aim of this paper is to determine the critical factors of information and communication technologies (ICT), which contribute to increasing the capabilities of ICT, and what to extent they contribute to improving the operational and financial performance of SMEs in commerce sector. With this purpose, the quantitative analysis is performed with the structural equation model a multivariate technique, which allows confirming whether the variables proposed in the model are related and in what degree they explain the phenomenon, with data provided by the companies surveyed from the SIEM.
\end{abstract}

Key words: Structural Equations, Performance, Information Technology, MSMEs.

JEL: M10, M15, M16.

Resumen: El presente artículo tiene como objetivo determinar los factores críticos de las tecnologías de la información y comunicación (TIC) que contribuyen a incrementar las capacidades de las TIC y en qué grado contribuyen estas a la mejora del desempeño operacional y financiero de las MIPyMES de comercio. Con este propósito se realiza el análisis cuantitativo con la técnica multivariante de ecuaciones estructurales la cual permite confirmar si las variables propuestas en el modelo se relacionan y en qué grado explican el fenómeno usando datos proporcionados por las empresas encuestadas del Sistema de Información Empresarial Mexicano (SIEM).

Palabras clave: Ecuaciones estructurales, Desempeño, Tecnologías de la información, MIPYMES.

Impacto de las capacidades de las TIC 
Palabras clave: Ecuaciones Estructurales, Desempeño, Tecnologías de la información, MIPyMES

\section{Introducción}

Las MIPyMES, constituyen la columna vertebral de la economía nacional, por su alto impacto en la generación de empleos y en la producción nacional. De acuerdo con datos del INEGI (2014), en México existen aproximadamente 4 millones 15 mil unidades empresariales, de las cuales $99.8 \%$ son MIPyMES que generan $52 \%$ del Producto Interno Bruto (PIB) y $72 \%$ del empleo en el país.

Según el censo económico del INEGI (2014), las MIPyMES en México son un eslabón fundamental, indispensable para el crecimiento del país. México cuenta con una importante base de estas empresas, claramente más sólida que muchos otros países del mundo, que debe ser aprovechada para hacer de ellas una fortaleza que haga competitivo al país. La relevancia de estas se refiere tanto a producto interno bruto, como a personal ocupado y a número de establecimientos según los resultados obtenidos en este censo económico.

En el caso de Nuevo Leon, el censo económico de INEGI (2014), las MIPyMES conforman más del $99 \%$ de las empresas del estado, estas empresas empelan el $58.8 \%$ de la población económicamente activa en comparación con las grandes empresas del estado que emplean al 41.2\%. De acuerdo con el Sistema de Información Empresarial Mexicano (SIEM)el $67 \%$ de las MIPyMES son empresas de comercio de las cuales el $66 \%$ se encuentran ubicadas en el área metropolitana de Monterrey. 
El propósito de esta investigación es determinar el impacto de las capacidades de las TIC en la mejora del desempeño financiero y operacional de las MIPyMES, la revisión de literatura sobre este tema sugiere que la mayor parte de las investigaciones hechas sobre el tema se desarrollaron en países desarrollados por lo que existe un área de oportunidad en este tema.

\section{Marco teórico}

La revisión de literatura previa sugiere que las TIC ofrecen nuevas oportunidades a las pequeñas empresas que les permiten compensar desventajas competitivas tales como tamaño, recursos y mercado (Jones, Motta y Alde, 2016; Regan y Wymer, 2011; Qureshil, Kamal y Wolcott, 2009).

Aunque existe literatura sobre el tema, la mayor parte de las investigaciones se ha realizado en países desarrollados y empresas grandes como Johnson y Johnson, Walmart, Dell (Tarutè y Gatautis, 2014; Fernandez, Ferreras, Alegre, y Chiva, 2014; Piget y Kossai, 2013; Chung, Byrd, y Lewis, 2005), por lo que existe un área de oportunidad para confirmar que capacidades de las TIC impactan el desempeño de las MIPyMES de comercio ya que la literatura en países en desarrollo como México es escasa.

El estudio de las capacidades de las TIC y su contribución a mejorar el desempeño operacional y financiero ha sido abordado desde diferentes perspectivas como: la teoría basada en el conocimiento (Alavi y Leidner, 2001; Perez-Lopez y Alegre, 2012), la teoría de la riqueza de

\section{Impacto de las capacidades de las TIC}


medios (Daft y Lengel, 1986; Banker, Bardhan y Asdemir, 2006) y la teoría basada en recursos (RBV).

La teoría RBV se considera como la base de la ventaja competitiva de una empresa, ya que el crecimiento de las empresas es motivado y a la vez limitado por la administración en su búsqueda de un uso óptimo de los valiosos recursos tangibles o intangibles de los que dispone la empresa (Moriones, Billon y Lopez, 2013, Barney, 1991). La revisión de literatura previa sugiere que desde la perspectiva RBV, la infraestructura, el conocimiento y las inversiones de TIC contribuyen a mejorar el desempeño (Ghobakhloo, Hong y Jabeen 2015; Palomo y Islas 2016). También bajo esta misma perspectiva (Lo y Leidner, 2018; Bharadwaj, 2000), sugiere que las capacidades, el gobierno y la estrategia son vistas como recursos clave que permiten obtener una ventaja competitiva sostenible que contribuya a mejorar el desempeño de las empresas.

Las capacidades de las TIC (TCAP) han sido estudiadas tomando en cuenta variables como: el conocimiento de las TIC, las operaciones de las TIC y la infraestructura de las TIC mediante la técnica de ecuaciones estructurales. Para el presente estudio se analizan variables como: infraestructura, inversiones financieras, estrategias de uso y el gobierno de las TIC, que son abordadas desde la perspectiva RBV (Perez-Lopez y Alegre, 2012).

De acuerdo con la revisión de literatura previa la capacidad de uso de TIC, se define como la forma en que las TIC influyen de manera positiva en los procesos de la empresa que permiten generar una ventaja competitiva para mejorar el desempeño del negocio y sugieren que los líderes en las empresas deben enfocarse en crear condiciones

\author{
Islas, J., Palomo
}


necesarias para el desarrollo de las TIC porque estas juegan un papel fundamental en la mejora del rendimiento del negocio (Chae, Kho, y Park, 2018; Perez-Lopez y Alegre, 2012; Mithas, Mithas, Sambamurthy, 2011; Wu, Sengun y Kim, 2006; Subramani, 2003). Para Fernandez et al., (2014), las capacidades de las TIC se refieren a la forma en como las empresas emplean las tecnologías para gestionar la información efectivamente, que satisfaga las necesidades de información de las empresas en forma de ventaja competitiva.

La infraestructura de TIC (TINF), generalmente es definida como todo lo relacionado con hardware y software, es decir bases de datos, sistemas de planeación de recursos, sistemas de gestión de clientes 0 telecomunicaciones (Suprapto, Husada y Basana, 2017; Rai, Patnayakuni y Patnayakuni 1997). Existe evidencia que relaciona la infraestructura de las TIC con la mejora en el desempeño de las empresas ya que el constructo infraestructura de TIC dentro de la empresa facilita la masificación y por ende tiene impacto en el desempeño del negocio (Chae et al., 2018; López y Muneta, 2011). Por ejemplo: Dell Computer Corporation y Walmart que elevaron su rentabilidad mediante el apoyo de su infraestructura tecnológica (Law y Ngai, 2007; Chung et al., 2005).

Medir los beneficios de las inversiones financieras en TIC (TINF) y su contribución a mejorar el desempeño es una de las principales preocupaciones de las MIPyMES (Johannessen, Olaisen, y Olsen, 1999). La literatura previa sugiere que el efecto de las TIC en el desempeño puede ser estudiado mediante indicadores como la eficiencia, crecimiento, nuevos productos, ventas, incremento en la cuota de mercado, nuevos mercados- entre otros (Abrego, Sánchez, y Medina,

\section{Impacto de las capacidades de las TIC}


2016; Moriones et al., 2013). Las inversiones financieras de TIC se definen como el presupuesto, gastos relacionados con las TIC (Fernandez et al, 2014; Li y Ye, 1999); en literatura previa se sugiere que inversiones de TIC tienen un impacto positivo en el desempeño de la empresa (Lee, Choi, Lee, Min, y Lee, 2016; Moriones et al., 2013).

La estrategia para la implementación de TIC (TSTR), es definida como el grado de proactividad en la planeación de estrategias de adopción uso y mejora de las TIC en la empresa (Lo y Leidner, 2018; Rivard, Raymond y Verreault, 2006). La estrategia para la implementación de TIC puede jugar un rol importante dentro de la organización y convertirse en un elemento importante cuando se definen las metas de la organización relacionadas con la eficiencia organizacional, los costos y la calidad como factores de gran significancia para la empresa (Lee et al., 2016; Haki y Forte, 2010; Johnson y Lederer 2010; Li y Ye 1999).

El Gobierno de TIC (TGOV), es definido como las políticas de gestión de las TIC, las empresas en general han desarrollado procesos que dependen cada vez más de las TIC. Literatura previa sobre el tema sugiere que el gobierno de TIC se ha convertido en un tema importante, ya que un Gobierno de TIC efectivo permite a las empresas crear y desarrollar capacidades de TIC, lo que a su vez da como resultado un mejor rendimiento de la empresa (Sirisomboonsuk, Ching, Qing \& Burns, 2018; Zhang, Zhao y Kumar, 2016).

Ghobakhloo et al. (2015), definen el desempeño del negocio como efectividad organizacional. El desempeño del negocio también es conocido como rendimiento del negocio. La revisión literaria identifica 
principalmente dos tipos de desempeño: el operacional y el financiero (Consoli, 2012; Santos y Brito, 2012; Liang, You, y Liu, 2010), por lo que la presente investigación, desempeño se referirá a estos dos tipos.

El desempeño financiero (TDESF) es representado generalmente como: la rentabilidad sobre inversiones, rentabilidad sobre recursos, las utilidades y las ventas, el valor de mercado de las empresas; estos indicadores usualmente comprueban si la empresa es capaz de generar ganancias (Shicheng, Lin, y Chou, 2018; Santos y Brito, 2012; Liang et al., 2010). El desempeño operacional (TDESO) es asociado con medidas como: costos, ahorros, calidad, reducción de tiempos, desarrollo de nuevos productos, así como la mejora en el servicio al cliente (Suprapto et al., 2017; Moriones et al., 2013; Lee, Chu y Tseng, 2011).

Con base en lo anterior la hipótesis que se plantea es la siguiente:

\section{Hipótesis}

Las inversiones financieras, las estrategias para implementación infraestructura, el gobierno y la infraestructura de las TIC; son los factores que contribuyen al desarrollo de nuevas capacidades, que impactan en el desempeño financiero y operacional de las MIPyMES de comercio localizadas en el área metropolitana de Monterrey. Se propone el siguiente modelo: 
Figura 1. Modelo estructural propuesto

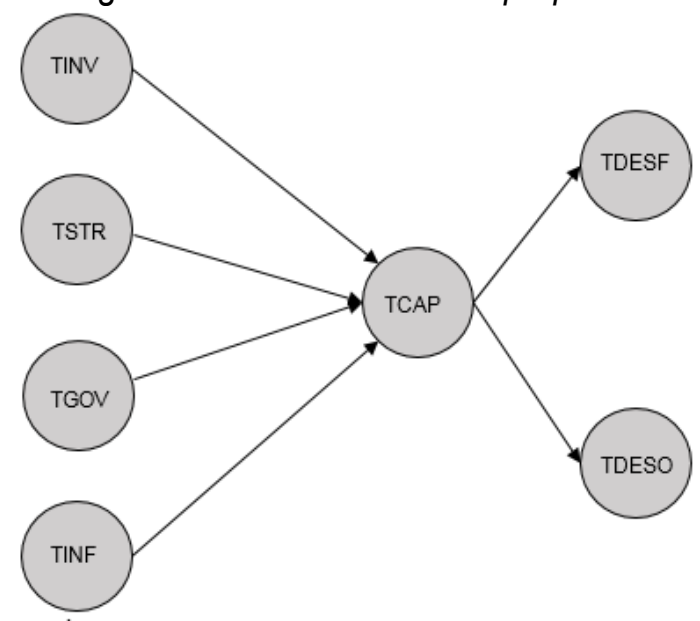

Fuente: Elaboración propia con Smart PLS 3.

\section{Metodología}

En el presente estudio se analizaron MIPyMES del área metropolitana de Monterrey mediante una encuesta realizada de manera electrónica en Google forms. Lo anterior se aplicó a empresas registradas en el SIEM con datos extraídos en noviembre del 2017. Se obtuvo respuesta de 51 empresas en un periodo de diciembre del 2017 a junio del 2018. De estas empresas 20 son micros, 14 pequeñas, 17 medianas. La encuesta fue aplicada al dueño, director general o gerente general de las empresas ya que son los responsables de tomar decisiones estratégicas y definir las 
políticas relacionadas con las TIC. Este estudio es exploratorio y tiene un diseño no experimental, correlacional-causal y explicativo.

Como inicio de la metodología se realizó un análisis factorial confirmatorio con la intención de validar el acomodo de los ítems en cada variable dentro del instrumento de medición, que será detallado en futuras investigaciones. Seguido del análisis factorial se procedió a realizar el análisis de Cronbach con finalidad de medir la confiabilidad de cada una de las variables incluyendo la dependiente.

Se pudo confirmar que los ítems que están cargando en cada una de las variables tienen la consistencia interna de acuerdo con las mediciones obtenidas en el indicador de alfa de Cronbach. En la tabla 1 es posible observar el resultado del análisis con las variables que más ítems fueron eliminadas de cada constructo de acuerdo con la metodología de alfa de cronbach.

Tabla 1. Alfa de Cronbach

\begin{tabular}{cccc}
\hline Variable & $\begin{array}{c}\text { No. } \\
\text { İtems } \\
\text { Origina } \\
\text { les }\end{array}$ & $\begin{array}{c}\text { Valor } \\
\text { Alfa }\end{array}$ & No. Ítems Final \\
\hline $\begin{array}{c}\text { Infraestructura de TIC (TINF) } \\
\text { Capacidades de uso de las TIC } \\
\text { (TCAP) }\end{array}$ & 7 & 0.827 & 5 \\
$\begin{array}{c}\text { Estrategia para la implementación } \\
\text { de TIC (TSTR) }\end{array}$ & 6 & 0.902 & 4 \\
$\begin{array}{c}\text { Inversiones financieras de TIC } \\
\text { (TINV) }\end{array}$ & 5 & 0.916 & 4 \\
\end{tabular}

Impacto de las capacidades de las TIC 


\begin{tabular}{cccc} 
Gobierno de TIC (TGOV) & 6 & 0.936 & 4 \\
Desempeño del negocio (TDESF) & 2 & 0.967 & 2 \\
Desempeño del negocio (TDESO) & 2 & 0.967 & 2 \\
Total & 33 & & 25 \\
\hline
\end{tabular}

Fuente: Elaboración propia, con SPSS 23.

Como se observa en la Tabla 1, se presenta el número de ítems originales y el número finales de cada variable. En total se eliminaron 8 ítems del instrumento de medición. Todas las variables perdieron ítems a excepción de inversiones financieras de TIC. El alfa de Cronbach para cada variable tiene un valor por arriba de 0.80 por lo que es confiable para el estudio que se realiza.

Análisis de Ecuaciones Estructurales

Con base en la información disponible para el estudio se hace viable la utilización del modelo de ecuaciones estructurales (SEM). Existen dos enfoques principales que son los basados en el análisis de covarianza con software como AMOS con muestras más grandes con rangos de 200-800 observaciones y el análisis de los mínimos cuadrados parciales (PLS) con SmartPLS 3 es la técnica estadística elegida para este estudio dado el número de observaciones con que se cuenta para este estudio ya que PLS requiere muestras menores (Schwarz, Kalika, Kefi y Schwarz, 2010).

La figura 2 muestra el modelado propuesto basado en ecuaciones estructurales, que como se puede observar las variables exógenas tales 
como: TINF, TSTR, TINV, TGOV, y las variables endógenas TDESF y TDESO que básicamente forman el modelo interior (inner model) y que junto con el modelo exterior (outer model), serán analizados mediante las variables latentes y sus ítems asociados a cada constructo.

Figura 2. Modelo de factores modelo exterior/modelo interior

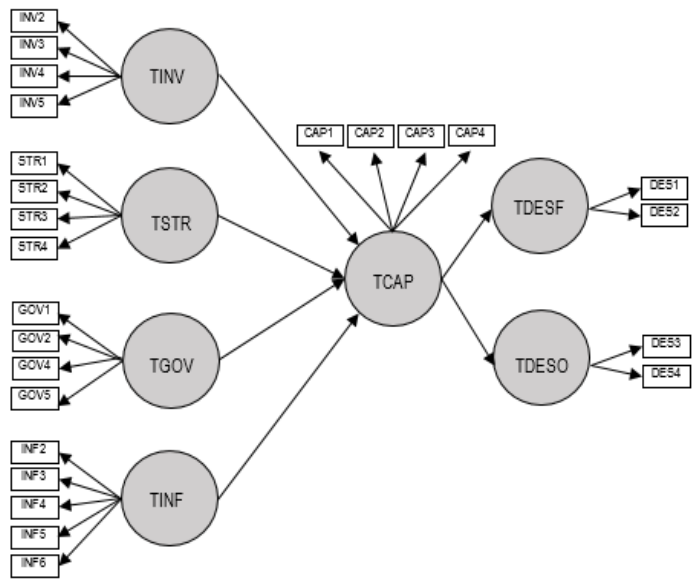

Fuente: Elaboración propia con Smart PLS 3.

La tabla 2 que se muestra a continuación muestra las cargas y los pesos (weigths/loadings) de cada de los ítems que componen los constructos del modelo de investigación, los límites de las cargas se deben de encontrar en el rango de mínimo 0.4 o mayor. Con este análisis es posible observar la confiabilidad de cada ítem representada con la carga en su respectivo constructo y se confirma que los ítems miden para lo que fueron diseñados los constructos (Schwarz et al., 2010).

Impacto de las capacidades de las TIC 
También en la misma tabla 2 se puede observar que existen algunos ítems que son menores a 0.4 como TSTR con alguno de los valores más bajos y negativo como el ítem TSTR3 con un valor de -0.150 . Así mismo podemos observar que los ítems de los constructos TCAP, TDES, TGOV, TNV están en su mayoría por arriba del valor de 0.4. Esto indica que los valores son adecuados y validan la confiablidad de estos constructos.

Tabla 2. Cargas y Pesos del Modelo Propuesto

\begin{tabular}{cccccc}
\hline Variable & Peso & Carga & Variable & Peso & Carga \\
\hline CAP1 & 0.265 & $\mathbf{0 . 9 6 4}$ & INF2 & 0.148 & $\mathbf{0 . 7 8 5}$ \\
CAP2 & 0.235 & $\mathbf{0 . 9 2 7}$ & INF3 & 0.278 & $\mathbf{0 . 8 7 2}$ \\
CAP4 & 0.275 & $\mathbf{0 . 9 6 5}$ & INF4 & 0.244 & 0.838 \\
CAP5 & 0.291 & $\mathbf{0 . 8 9 8}$ & INF5 & 0.376 & 0.917 \\
DES1F & 0.561 & $\mathbf{0 . 9 9 0}$ & INF6 & 0.126 & 0.724 \\
DES2F & 0.451 & $\mathbf{0 . 9 8 5}$ & INV2 & 0.177 & 0.895 \\
DES30 & 0.537 & $\mathbf{0 . 9 8 7}$ & INV3 & 0.208 & 0.953 \\
DES40 & 0.478 & $\mathbf{0 . 9 8 4}$ & INV4 & 0.161 & $\mathbf{0 . 9 6 3}$ \\
GOV1 & 0.584 & $\mathbf{0 . 9 8 3}$ & INV5 & 0.501 & 0.973 \\
GOV2 & 0.430 & $\mathbf{0 . 9 6 8}$ & STR1 & -0.541 & -0.307 \\
GOV4 & -0.044 & $\mathbf{0 . 7 3 1}$ & STR2 & 1.072 & $\mathbf{0 . 6 3 0}$ \\
GOV5 & 0.049 & $\mathbf{0 . 8 5 9}$ & STR3 & -0.471 & $-\mathbf{0 . 1 5 0}$ \\
& & & STR4 & 0.163 & $\mathbf{0 . 5 3 9}$ \\
\hline
\end{tabular}

Fuente: Elaboración propia, con SmartPLS 3.

El análisis mostrado en la tabla anterior, indica que las cargas de los ítems no se relacionan con otros constructos del modelo. Por lo que a continuación se puede observar la tabla 3 de validaciones cruzadas. El 
criterio para esta tabla es los valores de los ítems deben ser los mayores para el constructo que fueron diseñados que en cualquier otro constructo.

Lo anterior confirma lo encontrado en la tabla 3, que el constructo de TSTR sugiere que no cuenta con la validez y confiabilidad, ya que las cargas de algunos ítems del constructo son menores a las cargas cruzadas con otras variables. Mientras que las cargas de los ítems de TCAP, TDES, TGOV, TNV, TSTR son mayores en el constructo para el que fueron diseñados y validan que los constructos miden para lo que fueron diseñados.

Tabla 3. Cargas Cruzadas del Modelo Propuesto

\begin{tabular}{llllllll}
\hline & TCAP & TDESF & TDESO & TGOV & TINF & TINV & TSTR \\
\hline CAP1 & 0.964 & 0.529 & 0.647 & -0.326 & -0.453 & 0.135 & -0.420 \\
CAP2 & 0.927 & 0.462 & 0.566 & -0.203 & -0.446 & -0.039 & -0.426 \\
CAP4 & 0.965 & 0.658 & 0.695 & -0.106 & -0.404 & 0.208 & -0.298 \\
CAP5 & 0.898 & 0.742 & 0.761 & -0.153 & -0.326 & 0.256 & -0.305 \\
DES1 & 0.699 & 0.990 & 0.978 & -0.182 & -0.433 & 0.595 & -0.038 \\
DES2 & 0.562 & 0.985 & 0.932 & -0.062 & -0.367 & 0.570 & 0.022 \\
DES3 & 0.745 & 0.922 & 0.987 & -0.314 & -0.467 & 0.581 & -0.187 \\
DES4 & 0.663 & 0.992 & 0.984 & -0.165 & -0.456 & 0.564 & -0.043 \\
GOV1 & -0.229 & -0.117 & -0.230 & 0.983 & 0.118 & -0.591 & 0.055 \\
GOV2 & -0.169 & -0.150 & -0.261 & 0.968 & 0.049 & -0.539 & 0.037 \\
GOV4 & 0.017 & -0.371 & -0.371 & 0.731 & -0.034 & -0.825 & -0.274 \\
GOV5 & -0.019 & -0.282 & -0.342 & 0.859 & 0.009 & -0.789 & -0.103 \\
INF2 & -0.202 & -0.056 & -0.197 & 0.429 & 0.785 & -0.256 & -0.173
\end{tabular}




\begin{tabular}{llllllll} 
INF3 & -0.379 & -0.578 & -0.566 & -0.092 & 0.872 & -0.206 & -0.229 \\
INF4 & -0.332 & -0.417 & -0.436 & 0.011 & 0.838 & -0.329 & -0.016 \\
INF5 & -0.512 & -0.373 & -0.441 & 0.023 & 0.917 & -0.046 & -0.027 \\
INF6 & -0.172 & 0.023 & -0.076 & 0.341 & 0.724 & -0.137 & -0.212 \\
INV2 & 0.080 & 0.510 & 0.476 & -0.527 & -0.071 & 0.895 & 0.308 \\
INV3 & 0.094 & 0.622 & 0.606 & -0.567 & -0.275 & 0.953 & 0.369 \\
INV4 & 0.073 & 0.459 & 0.451 & -0.606 & -0.077 & 0.963 & 0.232 \\
INV5 & 0.225 & 0.593 & 0.596 & -0.539 & -0.255 & 0.973 & 0.283 \\
STR1 & 0.122 & 0.196 & 0.212 & 0.099 & -0.060 & -0.172 & -0.307 \\
STR2 & -0.242 & 0.146 & 0.069 & 0.155 & -0.185 & 0.132 & 0.630 \\
STR3 & 0.106 & 0.237 & 0.252 & 0.158 & -0.127 & -0.075 & -0.150 \\
STR4 & -0.037 & 0.306 & 0.235 & 0.103 & -0.151 & 0.252 & 0.539 \\
\hline
\end{tabular}

Fuente: Elaboración propia, con Smart PLS 3.

A continuación, se muestra la tabla 4, donde se puede observar el coeficiente de la confiabilidad compuesta, el promedio de la varianza extraída (AVE) y las correlaciones los constructos de las variables latentes. 
Tabla 4. Confiabilidad Compuesta y Correlaciones

\begin{tabular}{|c|c|c|c|c|c|c|c|c|c|}
\hline & $\begin{array}{l}\text { Com } \\
\text { p. } \\
\text { Conf. }\end{array}$ & AVE & TCAP & $\begin{array}{l}\text { TDES } \\
\mathbf{F}\end{array}$ & $\begin{array}{l}\text { TDES } \\
0\end{array}$ & $\begin{array}{l}\text { TGO } \\
\mathrm{V}\end{array}$ & TINF & TINV & TSTR \\
\hline TCAP & 0.967 & 0.881 & 0.939 & & & & & & \\
\hline TDESF & 0.988 & 0.976 & 0.646 & 0.988 & & & & & \\
\hline TDESO & 0.985 & 0.971 & 0.717 & 0.969 & 0.986 & & & & \\
\hline TGOV & 0.938 & 0.794 & -0.208 & -0.130 & -0.247 & 0.891 & & & \\
\hline TINF & 0.917 & 0.689 & -0.431 & -0.408 & -0.469 & 0.092 & 0.830 & & \\
\hline TINV & 0.972 & 0.896 & 0.158 & 0.591 & 0.581 & $\overline{0} .579$ & -0.210 & 0.946 & \\
\hline TSTR & 0.137 & 0.201 & -0.382 & -0.011 & -0.121 & 0.055 & -0.130 & 0.311 & 0.448 \\
\hline
\end{tabular}

Fuente: Elaboración propia, con Smart PLS 3.

Los resultados de la tabla anterior muestran que los valores de la confiabilidad compuesta mayores al 0.80 son confiables, como se observa las variables con excepción de TSTR que tiene un valor 0.137 , las demás variables tienen valores por arriba de 0.90 . Promedio de la varianza extraída 0 average extracted variance (AVE) el valor recomendable es mayor a 0.50 , la variable TSTR tiene el valor más bajo con 0.201 y TINF con un valor de 0.689 pero dentro del rango de aceptable, los valores más altos son los TDESO, TDESF y TINV que son arriba de 0.90 (Schwarz et al., 2010). También se muestran las correlaciones de un constructo en comparación con los otros constructos con valores de la tabla.

A continuación, el modelo de ecuaciones estructurales. 
Figura 3. Modelo de factores modelo exterior/modelo interior.

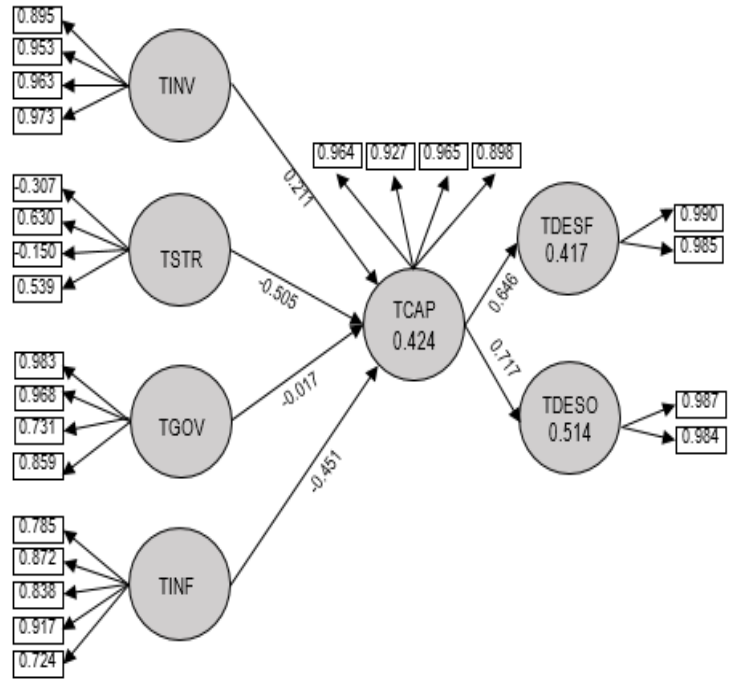

Fuente: Elaboración propia con Smart PLS 3.

El modelo anterior muestra las cargas de los ítems que componen cada constructo, así como los coeficientes estandarizados de cada variable, los que destacan más son los valores de las variable TGOV=0.017 que son menores a 0.20 en su valor absoluto que es el valore recomendado, por lo que estas variables no contribuyen a la explicación del fenómeno (Schwarz, 2010).

Las variables TINF=-0.451, TSTR $=-0.505$ son las que contribuyen a la explicación de TCAP con una relación inversa. Se puede observar que la variable TINV tiene el valor de coeficiente estandarizado de 0.211 
que es el mínimo recomendable y junto con TINF y TSTR son las variables que más contribuyen a la explicación de la variable endógena generando un valor de $\mathrm{R}^{2}$ de 0.424 .

También se puede observar el impacto de TCAP en las variables endógenas TDESF (0.646) y TDESO (0.717), que son valores por arriba del valor recomendado para los coeficientes estandarizados, y con $\mathrm{R}^{2}$ TDESF (0.417) y TDESO (0.514), es decir las capacidades tienen más impacto en el desempeño operativo que en el desempeño financiero.

\section{Conclusiones}

El estudio fue realizado en 51 MIPyMES del área metropolitana de Monterrey para determinar cuáles son las capacidades de las TIC que contribuyen a mejorar el desempeño financiero y operacional de las MIPyMES. Este tema es abordado desde la perspectiva basada en recursos ya que los factores que explican las capacidades de las TIC y las mismas capacidades de las TIC pueden ser vistos como recursos valiosos, raros, no imitables y no sustituibles que permiten conseguir una ventaja competitiva y contribuyen a incrementar el desempeño financiero u operativo de las empresas.

Como resultado del modelo propuesto se encontró como principal hallazgo que las variables TCAP, tiene una mayor explicación en el desempeño operacional (TDESO) con un coeficiente estandarizado de (0.717) y una $\mathrm{R}^{2}$ de (0.514). Este resultado sugiere que el desempeño debe ser medido de forma separada es decir financiero y operacional. Aunque existen estudios similares como (Fernandez et al., 2014; Perez y

\section{Impacto de las capacidades de las TIC}


Alegre, 2012). Estos solo abordan el desempeño como una sola unidad de medida.

También como hallazgo importante se observa el desempeño financiero con un coeficiente estandarizado de TDESF (0.646) y una $\mathrm{R}^{2}$ de (0.417). Estos valores reafirman que el desempeño debe ser abordado de manera independiente como sugiere (Consoli, 2010), aunque como se mencionó previamente, el desempeño o rendimiento normalmente es analizado como una sola unidad y no como sugiere el presente estudio.

Como se observa la figura 3 el coeficiente de determinación $\left(R^{2}\right)$ de TCAP es de 0.424, es decir las variables TSTR, TGOV, INV y TINF explican del $42 \%$ de la varianza. De acuerdo con Rositas (2014), en estudios experimentales se espera una $\mathrm{R}^{2}$ de 0.50 . Aunque el valor de $\mathrm{R}^{2}$ de TCAP está por debajo de valor sugerido, esta variable si tiene impacto en el desempeño operacional, ya que la $R^{2}$ de esta variable si está ligeramente arriba del límite sugerido.

Con respecto los factores que tienen impacto en las capacidades un hallazgo importante es que las TSTR y TINF tienen una relación inversa. Esto significa que entre más estrategias relacionadas con el uso de las TIC se definan en la empresa el impacto en las capacidades será menor, así como entre más infraestructura menos capacidades, esto puede ser atribuido al tamaño de las empresas ya que la falta de estrategias bien definidas podría afectar que se adquieran nuevas capacidades de las TIC.

TSTR, TGOV, INV y TINF no habían sido estudiadas en conjunto previamente como factores que impactan las capacidades de las TIC de las MIPyMES, pero si TINF que es abordada por diferentes autores y los

Islas, J., Palomo 
resultados no son consistentes con la literatura previa, ya que esta variable si es signifícate en estudios previos (Fernandez et al, 2014; Pérez \& Alegre, 2012). Con respecto TSTR el resultado si es consistente con la literatura previa revisada (Tarutè \& Gatautis, 2014).

Con respecto a TINV es el único factor que está dentro de los límites recomendados del coeficiente estandarizado con un impacto positivo de 0.21 en las capacidades, esta variable es consistente con los resultados de estudios previos (Moriones et al., 2013; Li \& Ye, 1999; Rai et al., 1997). Acerca de la variable TGOV es la variable que menos contribuye a la explicación de la variable endógena TCAP, este resultado no es consistente con estudios previos (Zhang et al., 2016).

\section{Contribución al conocimiento}

El estudio no se había realizado para este tipo de empresas, así como tampoco se habían analizado estos factores en conjunto, se validó que, de acuerdo con los resultados del estudio, las variables, TSTR, TGOV, INV y TINF pueden ser vistas como capacidades que contribuyen a mejorar el desempeño operacional de las MIPyMES, Es decir que estas empresas de comercio, deberían de enfocarse en desarrollar capacidades de TIC que contribuyan a mejorar la atención a clientes, la mejora de tiempos de entrega, control de costos o de calidad entre otros. También es importante poner especial atención a las estrategias y al gobierno de las TIC que se utilizan, ya que estas deben de estar alineadas con la misión y visión de la empresa, así como la infraestructura de TIC de la empresa dado que tener más infraestructura tecnológica no significa que las capacidades de las TIC mejoraran. Por lo que estos resultados

\section{Impacto de las capacidades de las TIC}


obtenidos pueden servir como base para que las MIPyMES que estén en la búsqueda de mejorar su desempeño financiero u operacional pongan especial atención en estos factores en conjunto y no de forma aislada también como aporte al conocimiento se encuentra la propuesta de medición de las variables de este estudio. Estos factores también servirán de base para que en investigaciones futuras se puedan identificar etapas o niveles de avance de las MIPyMES en el uso de las TIC, así como la identificación de prácticas relacionadas con TIC que contribuyen a mejorar el desempeño financiero y operacional de las empresas. También para futuras investigaciones es posible verificar si las capacidades de las $\mathrm{TIC}$ tienen impacto en temas específicos como la mejora en el servicio al cliente o la reducción de costos.

\title{
Referencias
}

Abrego Almazán, D., Sánchez Tovar, Y., \& Medina Quintero, J. M. (2017). Influencia de los sistemas de información en los resultados organizacionales. Contaduría y Administración, 62(2), 303-320. doi:10.1016/j.cya.2016.07.005

Alavi, M., \& Leidner, D. (2001). Review: Knowledge Management And Knowledge Management Systems: Conceptual Fundations And Research Issues. Management Information Systems Research Center, 25(1), 017-136. Disponible de http://www.jstor.org/stable/3250961

Banker, R. D., Bardhan, I., \& Asdemir, O. (Diciembre de 2006). Undesrtanding, The Impact of Collaboration software on Production Design and Development. Information Systems Research, 17(4), 352-373

Barney, J. (1991). Firm Resources and Sustained Competitive Advantage. Journal of Management, 17(1), 99-120

Bharadwaj, A. S. (Marzo de 2000). A Resource-Based Perspective on Information Technology Capability and Firm Performance: An Empirical Investigation.

\author{
Islas, J., Palomo
}


Management Information Systems Research, 169-196. Disponible en http://www.jstor.org/stable/3250983

Cataldo, A., McQueen, R., \& Sepúlveda, M. (2011). Big IT for small companies. Industrial Engineering, 48-54

Chae, H.-C., Kho, C. E., \& Park, K. O. (July de 2018). Information \& Management. Information technology capability and firm performance: Role of industry, 55(5), 525-546. doi: https://doi.org/10.1016/j.im.2017.10.001

Chung, S. H., Byrd, T. A., Lewis, B. R., \& Ford, F. N. (2005). An empirical study of the relationships between IT infrastructure flexibility, mass customization, and business performance. ACM SIGMIS Database, 36(3), 26-44. doi: $10.1145 / 1080390.1080394$

Consoli, D. (2012). Literature analysis on determinant factors and the impact of ICT in SME's. Procedia - Social and Behavioral Sciences, 62, 93-97. Obtenido de http://www.sciencedirect.com/science/article/pii/S187704281203457X

Daft, R. L., \& Lengel, R. H. (Mayo de 1986). Organizational Information Requirements, Media Richness and Structural Design. Management Science, 32(5), 554-571

Fernandez, M. A., Ferreras, J. M., Alegre, J., \& Chiva, R. (2014). IT competency and the commercial success of innovation. Industrial Management \& Data Systems, 114(4), 550-567

Gálvez, E., Riascos, S., \& Contreras, F. (2014). Influencia de las tecnologías de la información y comunicación en el rendimiento de las micro, pequeñas y medianas empresas colombianas. Estudios Gerenciales, 30(133), 355-364. doi: https://doi.org/10.1016/j.estger.2014.06.006

Ghobakhloo, M., Hong, T. S., \& Jabeen, S. (2015). IT Resources, IT-Enabled Capabilities, and Business Performance. Encyclopedia of Information Science and Technology, 4129-4139. doi: 10.4018/978-1-4666-5888-2.ch406

Haki, M., \& Forte, M. (2010). Proposal of a service oriented architecture governance model to serve as a practical framework for business-IT alignment. New Trends in Information Science and Service Science th $^{\text {th }}$ International Conference, 410417

\section{Impacto de las capacidades de las TIC}


Holsapple, W. C., \& Wu, J. (2011). An elusive antecedent of superior firm performance: The knowledge management factor. Decision Support Systems, 52, 271-283

Instituto Nacional de Estadística y Geografía. (2014). Censos Económicos 2014. Obtenido de www.inegi.org.mx: http://www.inegi.org.mx/est/contenidos/espanol/proyectos/censos/ce2014/

Johannessen, A., Olaisen, J., \& Olsen, B. (1999). Strategic use of information technology for increased innovation and performance. Information Management \& Computer Security, $\quad 7(1), \quad 5-22 . \quad$ doi: http://dx.doi.org/10.1108/09685229910255133

Johnson, A. M., \& Lederer, A. L. (2010). CEO/CIO mutual understanding, strategic alignment, and the contribution of IS to the organization. Information \& Management, 47(3), 138-149. doi:10.1016/j.im.2010.01.002

Jones, C., Motta, J., \& Alde, M. V. (2016). Gestión estratégica de tecnologías de información y comunicación y adopción del comercio electrónico en Mipymes de Córdoba, Argentina. Estudios Gerenciales, 32(138), 4-13. doi:https://doi.org/10.1016/j.estger.2015.12.003

Law, C., \& Ngai, E. (2007). IT Infrastructure Capabilities and Business Process Inprovements. Information Resources Management Journal, 20 (4), 25-47

Lee, H., Choi, H., Lee, J., Min, J., \& Lee, H. (2016). Impact of IT Investment on Firm Performance Based on Technology IT Architecture. Procedia Computer Science, 91, 652-661. doi: https://doi.org/10.1016/j.procs.2016.07.164

Lee, Y.-C., Chu, P.-Y., \& Tseng, H.-L. (2011). Corporate performance of ICT-enabled business process re-engineering. Industrial Management \& Data Systems, 111(5), 735-754. Obtenido de: http://dx.doi.org/10.1108/026355711111137287

Li, M., \& Ye, R. L. (Diciembre de 1999). Information technology and firm performance: Linking with environmental, strategic and managerial contexts. Information \& Management, 35(1), 43-51. doi:10.1016/S0378-7206(98)00075-5

li, Z., \& Yao, J. (2018). Testing for heteroscedasticity in high-dimensional regressions. Econometrics and Statistics. doi: https://doi.org/10.1016/j.ecosta.2018.01.001 
Liang, T.-P., You, J.-J., \& Liu, C.-C. (2010). A resource-based perspective on information technology and firm performance: a meta analysis. Industrial Management \& Data Systems, 110(8), 1138-1158

Lo, J., \& Leidner, D. (May de 2018). Are Dynamic Capabilities the Missing Link Between the IS Strategy and Performance Relationship?: A Model and Exploratory Test at Three Levels of Environmental Dynamism. Information Systems, 49(2), 3553. doi:10.1145/3229335.3229339

Lopez, O. A., \& Muneta, A. M. (2013). ICT Impact on competitiveness, innovation and environment. Telematics and Informatics, 204-210

Mithas, Ramasubbu, \& Sambamurthy. (2011). How Information Management Capability Influences Firm Performance. MIS Quarterly, 35(1), 237. doi:10.2307/23043496.

Moriones, A. B., Billon, M., \& Lopez, F. L. (2013). Perceived performance effects of ICT in manufacturing SMEs. Industrial Management \& Data Systems, 113(1), 117135.

Palomo, M. A., \& Islas, J. A. (Nov de 2016). Los factores competitivos que aportan las tecnologías de la información y comunicaciones para las MIPyMES. Factores Explicativos de Competitividad Empresarial, 1-17

Perez-Lopez, S., \& Alegre, J. (2012). Industrial Management \& Data Systems. Information techonology competency, knowledge process and firm performance, 112(4), 644-662. doi:10-1101/02635571211225521

Piget, P., \& Kossai, M. (2013). The Relationship between Information and Comunication Technology Use and Firm Performance in Developing Countries: A Case Study of Electrical and Electronic Goods Manufacturing SMEs in Tunisia. African Development Review, 25(3), 330-343

Qureshil, S., Kamal, M., \& Wolcott, P. (June de 2009). Information technology interventions for growth and competitiveness in micro-enterprises. International Journal of Enterprise Information Systems, 5(2), 72

Rai, A., Patnayakuni, R., \& Patnayakuni, N. (1997). Technology Investment and Business Performance. Comunications of the ACM, 40(7), 89-97

\section{Impacto de las capacidades de las TIC}


Regan, E., \& Wymer, S. (January-March de 2011). Influential factors in the adoption and use of e-business and e-commerce information technology (EEIT) by small \& medium business. Journal of Electronic Commerce in Organizations, 56. Obtenido de http://www.idea-group.com

Rivard, S., Raymond, L., \& Verreault, D. (2006). Resource-based view and competitive strategy: An integrated model of the contribution of information technology to firm performance. The Journal of Strategic Information Systems, 15(1), 29-50. doi: 10.1016/j.jsis.2005.06.003

Santos, J. B., \& Brito, L. A. L. (2012). Toward a subjective measurement model for firm performance. BAR - Brazilian Administration Review, 9(spe), 95-117. doi: 10.1590/s1807-76922012000500007

Schwarz, A., Kalika, M., Kefi, H., \& Schwarz, C. (2010). A Dynamic Capabilities Approach to Understanding the Impact of IT-Enabled Businesses Processes and ITBusiness Alignment on the Strategic and Operational Performance of the Firm. Communications of the Association for Information Systems, 26. doi:10.17705/1cais.02604

Shicheng C, P., C, Y. D., Lin, S.-C., \& Chou, C. S. (2018). Toward an IT investment decision support model for global enterprises. Computer Standards \& Interfaces, 59, 130-140. doi:https://doi.org/10.1016/j.csi.2018.04.001

Sirisomboonsuk., P., Ching, V., Qing, R., Burns, J. (2018). Relationships between project governance and information technology governance and their impact on project performance. International Journal of Project Management, 36(2). 287300. Disponible en: https://doi.org/10.1016/j.jproman.2017.10.003

Subramani, M. R. (Semptiembre de 2003). How Do Suppliers Benefit from it Use in Supply Chain Realtionship. Management Information Systems Research Center, 28(1), 45-73

Suprapto, W., Tarigan, Z. J. H., \& Basana, S. R. (2017). The influence of ERP system to the company performance seen through innovation process, information quality, and information sharing as the intervening variables. Proceedings of the 2017 International Conference on Education and Multimedia Technology ICEMT'17. doi:10.1145/3124116.3124131

\author{
Islas, J., Palomo
}


Tarutè, A., \& Gatautis, R. (2014). ICT impact on SMEs performance. Procedia - Social and Behavioral Sciences, 110, 1218-1225. Recuperado el 23 de 09 de 2014, de http://www.sciencedirect.com/science/article/pii/S1877042813056085

Wu, F., Sengun, Y., \& Kim, D. C. (2006). The impact of information technology on suply chain cpabilities and firm performance: A resource-based view. Industrial Marketing Management, 35, 499-504

Zhang, P., Zhao, K., \& Kumar, R. L. (2016). Impact of IT Governance and IT Capability on Firm Performance. Information systems management, 33(4), 357-373. Disponible en: http://dx.doi.org/10.1080/10580530.2016.1220218 